\title{
¿Por qué hablar \\ de currículo en la \\ educación inicial?
}

Volumen 6 N. ${ }^{\circ} 48$ enero-junio de 2020

ISSN: 0122-4328

ISSN-E: 2619-6069

pp. $79-89$

Why Talk about

Curriculum in Early

Education?
Por que falar

sobre currículo na

educação inicial?

Graciela Fandiño Cubillos*

Fecha de recepción: 28-02-20

Fecha de aprobación: 13-05-20

PARA CITAR ESTE ARTÍCULO

Fandiño, G. (2020). Por qué hablar de currículo en la educación

inicial. Nodos y Nudos, 6(48). https://doi.org/10.17227/nyn.

vol6.num48-11364

Profesora Universidad Pedagógica Nacional. 


\section{RESUMEN}

Este artículo de reflexión busca mostrar que plantear currículos para la educación de los niños y niñas de 0 a 6 años no implica escolarizar, como algunos creen, y que, para debatir sobre los qué y cómo de nuestra educación inicial, es necesario la formulación de currículos con enfoques que partan de las características de los niños de estas edades. Se presenta, entonces, una breve historia de las instituciones de educación inicial que muestran que este ha sido un debate central, luego se ahondará en los conceptos de currículo y, por último, se mostrarán algunos elementos del estado de cuestión en nuestro país y en la ciudad de Bogotá. Lo anterior sin desconocer que las ideas sobre esta etapa educativa están determinadas por las concepciones de niño o niños, por las condiciones sociales, políticas y culturales de la sociedad y, en especial, por la situación de las mujeres en ella.

Palabras clave: educación inicial; educación preescolar; currículo en educación inicial

\section{ABSTRACT}

This reflection article seeks to show that proposing curricula for the education of children from 0 to 6 years old does not imply schooling, as some believe, and that, in order to debate the what and how of our initial education, it is necessary to formulate curricula with approaches that start from the characteristics of children of these ages. Thus, a brief history of initial education institutions is presented, showing that this has been a central debate. Then it will delve into the concepts of curriculum and, finally, some elements of the state of question in our country and in the city of Bogotá will be shown. The above without ignoring that the ideas about this educational stage are determined by the conceptions of child or children, by the social, political, and cultural conditions of society and, especially, by the situation of women in it.

Keywords: initial education; preschool education; curriculum in initial education

\section{RESUMO}

Este artigo de reflexão busca mostrar que propor currículos para a educação de crianças de 0 a 6 anos não implica ir à escola (escolaridade), como acreditam alguns, e que, para debater o que e como da nossa formação inicial, é preciso formular currículos com abordagens que partem das características das crianças dessas idades. Apresenta-se um breve histórico das instituições de educação inicial, mostrando que este tem sido um debate central, a seguir se aprofundará nos conceitos do currículo e, por fim, serão apresentados alguns elementos da situação em nosso país e na cidade de Bogotá. 0 exposto sem ignorar que as ideias sobre essa fase educacional são determinadas pelas concepções de criança ou crianças, pelas condições sociais, políticas e culturais da sociedade e, principalmente, pela situação das mulheres nela.

Palavras-chave: educação inicial; educação pré-escolar; currículo na educação inicial 


\section{Algo de historia sobre las instituciones de educación inicial}

Ante todo, es importante recordar que la necesidad de la "educación" de los niños menores de 6 o 7 años está planteada desde los griegos. Historiadoras de la educación infantil, como Carmen Colmenar (1995), afirman que el mismo Aristóteles hablaba de la necesidad de una educación en el seno de la familia basada en el juego desde los 2 o 3 años, la cual debería complementarse, a partir de los 5 años, con la asistencia a la escuela; idea que compartieron varios de los pensadores de la educación (Quintiliano, 30-90 C.; Vives, 1493- 1549; Erasmo, 1466-1536). Los grandes pedagogos como Comenio (1592-1670), Rousseau (17121778) y Pestalozzi (1746-1827) también se refirieron de una manera especial a la importancia de la educación de los niños menores de 6 años en el hogar.

Ahora bien, para hablar de las instituciones para la educación de la primera infancia se retomarán las 3 etapas históricas a las que se refieren Sanchidrian y Ruiz (2010), aclarando que estas no son sucesivas, sino que se mantienen o se superponen en el tiempo.

Etapa pre-pedagógica. Los historiadores coinciden en afirmar que las instituciones de educación para los niños de primera infancia se crearon a partir de la primera industrialización (a finales del siglo XVIII y comienzos del XIX) para atender a los hijos de las trabajadoras de los sectores pobres. Estas instituciones tenían un carácter marcadamente asistencial y solo accidentalmente instructivo, y estaban dedicadas a la guarda y la atención de las necesidades básicas. Entre sus denominaciones están: "Salas de asilo", "Casas guardianas", Dame Schools. Sobre la función instructiva dice Colmenar (1995): "En cuanto a la débil enseñanza que en estos lugares se seguía, consistía fundamentalmente en el aprendizaje de oraciones y prácticas religiosas acompañadas de juegos - más bien sedentarios- y cantos" (p. 20). Además, las personas que atendian a los niños no tenían ninguna preparación para ello.

En la segunda etapa se ubican las instituciones que, si bien cumplian funciones de cuidado, poseen ya un carácter abiertamente educativo. Sobre ellas se producirán escritos que servirán para su difusión.
También, surge la preocupación por la formación de las personas, en su mayoría mujeres, que trabajarian con los niños. Dentro de estas instituciones se mencionan como pioneras las "escuelas de calceta", creadas en 1770 en Francia por J. F. Oberlin (1740-1826), quien se ocupó de la formación de sus maestras, a quienes llamó "conductoras de infancia". En estas escuelas "los menores solian jugar, sobre todo, mientras que los más mayores aprendian canto, dibujo, geografía, historia de las plantas, ejercicios religiosos, coser, hilar, hacer punto de media, etc." (Colmenar, 1995, p. 21).

Luego, se reconocen las instituciones creadas por el inglés Robert Owen (1771-1858), dentro de la fábrica donde era socio. En 1816 crea la primera escuela infantil (Infant School) para niños entre 1 y 6 años. Estas escuelas, que después fueron desarrolladas por Wilderspin, sufrieron cambios en sus contenidos, como afirma Sanchidrían (2010): "Wilderspin a diferencia de Owen, intentó adaptar el currículo de la enseñanza primaria a las capacidades de los niños más pequeños, por lo que su sistema se basaba en libros, lecciones, aparatos y aprender a fuerza de repetir" (p. 57). Wilderspin escribió varios manuales dirigidos a los maestros, que influyeron en la creación de escuelas infantiles en varios países europeos a principios del siglo XIX.

Una tercera etapa se ubica hacia la segunda mitad del siglo XIX con la creación de los Kindergarten por Federico Frobel (1782-1852), pedagogo alemán, discípulo de Pestalozzi y considerado el pedagogo de la educación infantil. Sus planteamientos pedagógicos a nivel teórico y práctico producirán el gran cambio en la educación infantil, ya que reconoce la especificidad de los niños de la primera infancia. La fundación, en 1840, del primer Kindergarten en Blanquenburgo (Alemania) será un hito histórico. "La educación basada en el juego, en el desarrollo natural de las potencialidades humanas del niño $y_{1}$ en definitiva, de su libre espontaneidad, fueron las premisas pedagógicas básicas que configuraron una nueva escuela de párvulos" (Colmenar, 1995, p. 25). Estas instituciones, que sufrieron persecuciones de indole política, posibilitarán otra concepción de educación infantil que inspirará a instituciones a las cuales asistirán, además, niños de clases medias, tanto en países de Europa como en Estados Unidos. 
El caso francés tal vez sea el que mejor nos ilustre esta nueva etapa. Si bien las primeras instituciones francesas denominadas "salas de asilo" estaban inspiradas en las Infant Schoo/inglesas, ya desde mediados del siglo XIX, por la influencia de las ideas de Frobel, las pedagogas francesas Pape-Carpantier (1815-1878) y Pauline Kergomard (1838-1925), crearian la Ilamada "Escuela Maternal" francesa, tan valorada en este pais. Estas instituciones, como su nombre lo dice, son "escuela" en el sentido de que son una institución con una orientación claramente educativa, y a su vez es maternal, pues contempla las necesidades de cuidado de los niños de estas edades. Pero lo más diferenciador es que, si bien su origen está en las instituciones religiosas y filantrópicas, a mediados del siglo xIx empiezan a ser asumidas y reguladas por el estado.

La siguiente cita que Michelet (1988) toma de Kergomard muestra el debate que, ya en los albores del siglo $x x$, se da entre una mirada que Sanchidrian llama "primarizante"1 y una que respeta las características del niño: "la escuela maternal se ha ido desviando poco a poco de sus fines y se ha visto desbordada por la enseñanza primaria, se olvida que tiene un objetivo propio, que no ha de ser ni guardería, ni escuela elemental" (p. 228).

La anterior cita evidencia el debate que, según los autores, estuvo vigente durante el siglo $x x$-que es cuando se dará la expansión de este nivel educativo, con la llegada casi masiva de las mujeres al trabajo fuera del hogar ${ }^{2}$ - y que aún lo está en Colombia y en América Latina en el siglo XXI, este es: si la educación de los niños de primera infancia debe ser primarizante, o debe más bien respetar las características del niño y desde estas tener su propio objetivo educativo. Recordemos que la superación del "asistencialismo" se hizo bajo los contenidos de la educación primaria y pasará algún tiempo para que la educación sea pensada desde las particularidades de los niños de 0 a 6 años.

1 Término que se usará en este escrito, reemplazando el de "escolarización", pues al hablar de instituciones educativas estamos hablando de escuela. Se distinguirá entre "primarización" para referirnos a los enfoques centrados en la preparación para la primaria y "potenciamiento del desarrollo" para los que se centran en las caracteristicas de los niños en estas edades.

2 Serán las mujeres las que necesitan, o necesitamos, de unas instituciones de calidad, que procuren las mejores condiciones para el óptimo desarrollo de nuestros niños y niñas.
Ahora bien, es indudable el gran desarrollo teórico-práctico que tuvo la educación de la primera infancia desde los inicios del siglo xx, especialmente en la perspectiva más centrada en las características del niño. Pedagogas y pedagogos como Montessori, las hermanas Agazzi y Decroly, considerados como pedagogos del movimiento de Escuela Nueva o Activa, entrarán a robustecer las ideas de Froebel. Pero será el movimiento en general el que aportará al avance de la educación de la primera infancia, sin desconocer que este se desarrolló fundamentalmente para la educación primaria, que en la gran mayoría de paises europeos era ya obligatoria.

Luzuriaga (1943) plantea que las grandes ideas de la Escuela Nueva son: actividad, vitalidad, libertad, individualidad y colectividad. Estas ideas irán enriqueciéndose durante el siglo xx con los nuevos aportes de la psicología genética o piagetiana, el psicoanálisis y la biología. Se hablará de un niño activo, no solo que se mueve y hace actividades manuales sino como un activo constructor de sentido. Que la escuela se debe incorporar a la vida y el medio vital, social y cultural donde se encuentran los niños. La libertad de movimiento y actividad como principio fundamental, libertad de exploración, de expresión, sin duda de juego y de elección. Lo anterior permitirá la expansión de la creatividad, de la imaginación, de la solución de problemas. La individualidad hará énfasis en las particularidades de cada uno y sobre todo en el respeto a las diferencias individuales y la colectividad que es la base de la escuela, ya que alli se aprende a vivir con otros y se propende por la convivencia, la cooperación y el trabajo en grupo.

En Colombia, lamentablemente, tenemos muy pocos estudios históricos sobre las instituciones educativas para la primera infancia. ${ }^{3}$ El Ministerio de Educación Nacional (MEN) (1996), basándose en particular en Cerda (1982), periodiza la educación preescolar en 4 momentos: un primer momento, desde los inicios del siglo xx hasta finales de la década del 30 y habla de un modelo asistencial. Un segundo momento, de 1931 a 1975, se refiere a dar mayor énfasis

3 Algunos estudios se están realizando en la maestría de educación de la Universidad de Antioquia. 
al aspecto educativo. Un tercer momento: la aparición de un modelo curricular. Y el cuarto momento, a partir de la constitución de 1991.

En esta periodización no se hace un mayor énfasis en las diferencias de las etapas, en tanto se afirma que: aunque los modelos de atención han sido diferentes "coinciden en la búsqueda de metodologías que propenden por el desarrollo integral y armónico de los niños y la vinculación de la familia al proceso educativo" (Men, 1996, p. 14). Lo anterior nos muestra una de las características de nuestra mirada a la educación inicial, y es el poco debate con el que la asumimos, se pensaría que todo nos parece bien. Desde luego, nadie puede negar las buenas intenciones que se tienen, y en una perspectiva de diálogo y debate es imprescindible partir de estas, ya que en ellas hay creencias y saberes. Pero para ir construyendo nuevas miradas es necesario profundizarlas, explicitarlas y sobre todo debatir las existentes. Por ejemplo, en Colombia hay un "imaginario" generalizado de que las instituciones de bienestar, que atienden a los niños de los sectores populares, tienen un enfoque primordialmente asistencialista y que las atendidas o regidas por el Ministerio son educativas. Sin embargo, los documentos oficiales confrontan ese imaginario.

\section{El campo del currículo}

El temor que existe por parte de algunas maestras y especialistas de que exista un currículo para la educación inicial es, en principio, alentador porque, de cierta manera, quiere decir que creemos que los niños menores de 6 años no deben ser "primarizados", premisa que, desde luego, se comparte. Sin embargo, se plantea que hablar de currículo para el caso de la educación de los niños y niñas de primera infancia no implica lo anterior. En la medida en que se acepta el carácter educativo en esta etapa, se reconoce que tiene un currículo, y esto lleva a tener un proyecto formativo como lo explica Zabalza (2008): "Proyecto en cuanto es pensado, planificado, y de ahí se hace visible, público y compartido y Formativo porque está vinculado a la potenciación de un desarrollo equilibrado en todas y cada una de las dimensiones del niño" (p. 3, énfasis propio).
Ahora bien, en este debate es importante aceptar que el concepto de currículo no es de nuestra tradición. Nuestra tradición educativa viene más de Francia y Alemania, y los conceptos más cercanos son el de "pedagogía" y "didáctica". El término currículo es de la tradición anglosajona y llegó al país en la década de los 70 del siglo Xx, con la llamada "expansión educativa" en el gobierno de López Michelsen (19741978), en la cual se implementó la doble jornada en los colegios oficiales y, además, estuvo acompañada por la preocupación por la calidad educativa.

La calidad de la educación vino de la mano con la generalización para América Latina de los modelos de la tecnología educativa, desarrollados a partir de las "teorías conductistas" o del Análisis Experimental de la Conducta, en boga en Estados Unidos. De hecho, en 1976 se expide el Decreto 088 que reestructura el Ministerio de Educación y, además de incluir el preescolar como el primer nivel de educación formal, crea la Dirección General de Capacitación y Perfeccionamiento Docente, Currículo y Medios Educativos. Esta dirección se encargó de realizar currículos para todos los niveles y áreas del sistema educativo y se llamó la "Renovación Curricular". Dentro de esta se produce el Currículo de Preescolar (niños de 4 a 6 años) entre los años de 1977 a 1987. Los currículos de la renovación curricular se expidieron por el Decreto 1002 de 1984.

Este surgimiento llegó, no sin serios debates. Entre los investigadores que han indagado sobre el tema, se menciona a Aristizábal (2012) quien resume una posición crítica así:

El propósito de los decretos en el caso de la educación básica y media era desarrollar la estrategia de renovación curricular. Su sentido era cumplir con directrices internacionales, concretamente para América Latina, que urgían por una reorganización de los sistemas educativos procurando que se hablara el mismo lenguaje: el del diseño instruccional como núcleo de un modelo curricular, el de objetivos, un diseño detallista, centralizado, controlador del quehacer docente y a la larga inhibidor de la creatividad, que ofrecia la "virtud" de prefijar todo desde las oficinas de los expertos, para que otros -las/ los docentes- lo ejecutaran, en lo posible con el máximo de precisión. (p. 29) 
Sin embargo, aunque esta crítica esté sustentada no se puede desconocer, como lo menciona el profesor Vasco (2011), que la crítica a la tecnología educativa y al diseño instruccional estuvo en el mismo Ministerio, ya que existían funcionarios y asesores que venían de otras tradiciones -especialmente de la piagetiana- en oposición abierta con el conductismo, como es el caso del Profesor Federicci, asesor de los grupos de matemáticas, quien será luego reemplazado por el mismo profesor Vasco.

Vasco (2011) menciona tres documentos que se realizaron sobre los fundamentos de la renovación curricular: los epistemológicos, los psicológicos y los pedagógicos, e invita a revisarlos para comprobar cómo incluyeron en ellos el debate en el que el mayor problema fue el de psicología, por el tema del aprendizaje, y en donde el Análisis Experimental de la Conducta tiene una posición que contrasta con la piagetiana: "Al final se optó por poner en paralelo los cuatro modelos en conflicto, para que los maestros escogieran el que más les convenciera: el modelo de Piaget, el modelo de Skinner, el de Bandura y el de Gagné (pp. 42-44)" (p. 26).

El concepto de currículo se profundiza de la mano de Díaz Barriga (2003), quien hace un análisis histórico de lo que llama el campo del currículo. De una parte, plantea que el concepto de currículo surge en los Estados Unidos para atender la educación del hombre de la era industrial, dentro del sistema educativo organizado por el Estado. Pero desde alli nació con dos vertientes, una vinculada a los procesos educativos, las experiencias y el desarrollo de cada estudiante (alli estaría Dewey con su obra "El niño y el currículo") y otra a la necesidad de establecer con claridad una secuencia de contenidos que fundamenten la elección de los temas de enseñanza, ligada a las instituciones. La tendencia que se generalizó fue la segunda y es la que llegó a América Latina en la década de los 70 del siglo xx. Pero a finales de esta década, y en especial en los años 80 , resurgirá la perspectiva de la experiencia, ligada a lo que acontece en las instituciones educativas, con la incursión de diferentes disciplinas como la sociología, la antropología, incluso todas las miradas críticas de la escuela.
Esto producirá una generalización del campo de currículo, tanto en Europa como en América Latina.

Así es como emerge en el estudio del currículo una dimensión procesual (Gimeno, 1998). Esta perspectiva dinamiza el pensamiento sobre el currículo, que pasa de entenderlo como un plan que después se plasmará en la realidad, a pensarlo como un proceso en el que ocurren múltiples transformaciones que le dan unos particulares sentidos y significados.

Gimeno (1998) hablará del currículo como un proceso social, que se crea y deviene experiencia a través de múltiples contextos que interaccionan entre sí, es decir, el currículo como concurrencia de prácticas. Entre los currículos más tangibles menciona: El currículo prescrito y regulado, como el compendio de contenidos ordenados en las disposiciones administrativas. El currículo diseñado, creado para ser consumido por profesores y alumnos: los libros de texto, o materiales diversos. El currículo organizado, o sea, las programaciones o planes que hacen las instituciones educativas. El currículo en acción, o el conjunto de tareas de aprendizaje que realizan los alumnos que son, en parte, regulados por las programaciones del profesor y El currículo evaluado, como lo que los profesores exigen en sus exámenes y la manera como lo valoran (Gimeno, 1998, pp. 119-128).

Para precisar lo anterior, se hará referencia al caso de la educación inicial en el país, orientado por la pregunta: ¿el hecho de no hablar de currículo hace que no haya currículos? Si se toma lo expuesto arriba, se afirmará: donde hay escuela hay currículo, no hay escuela sin currículo. En este sentido, si se acepta que la educación inicial se hace en la escuela, en ella exis-

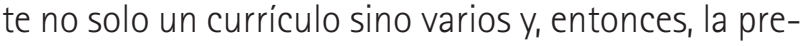
gunta es más bien: ¿qué tan primarizantes o qué tan respetuosos de las características de los niños de estas edades son los currículos para la educación inicial en nuestro país? Para seguir profundizando en este debate, vale la pena retomar la historia del currículo prescrito de la educación de los niños menores de 6 años en Colombia, tanto en el Ministerio (men) como en el Instituto Colombiano de Bienestar Familiar (ICBF). 


\section{Algo de historia en los currículos de la educación inicial en Colombia}

Aunque se reconoce que es en 1976 cuando por primera vez la Educación Preescolar forma parte del sistema educativo, ya en ese año hay 48 Jardines Infantiles Nacionales, de los cuales habría que indagar sobre sus planes de estudio y sus prácticas. Sin embargo, es dentro de la citada Renovación Curricular donde se elaboró el primer currículo para la educación de los niños de 4 a 6 años. Su primera versión se publicó en 1984 y luego fue ajustado y complementado en 1987. El documento partió de una información sobre la realidad o sobre el currículo en acción de jardines públicos y privados, que se describe de la siguiente manera:

Se observó que cada jardín empleaba su propio programa: algunos utilizaban programas traídos de realidades diferentes a la colombiana; otros tomaban la cómoda posición de dejar hacer a los niños lo que quisieran; la gran mayoria intentaban llenar a los niños de muchos conocimientos transmitidos verticalmente, limitándose de esta manera el necesario juego libre; otros se dedicaban únicamente a la expresión artística. Casi todos desconocian la realidad socioeconómica y su incidencia en el desarrollo de los niños. (Men, 1984, p. 19)

Ahora bien, en el Decreto 1002 de 1984, que reglamenta los planes de estudios elaborados en la Renovación Curricular, se encuentra como objetivo de la educación preescolar:

Desarrollar integral y armónicamente sus aspectos: biológico, sensomotor, cognitivo y socioafectivo, y en particular la comunicación, la autonomía y la creatividad, y con ello propiciar un aprestamiento adecuado para su ingreso a la Educación Básica

En el documento se plantean cuatro formas de trabajo: 1) El Trabajo comunitario, dirigido a la familia y a la comunidad para posibilitar un ambiente que favorezca el desarrollo integral del niño. 2) El Juego libre, como una forma de trabajo, en la que el maestro dispone diversos ambientes de juego entre los cuales el niño elige con libertad. Dentro de los materiales para el juego libre están: los creativos expresivos, que incluyen todas las artes y literatura; los de desarrollo cognitivo que ejercitan funciones del pensamiento (materiales didácticos) y materiales que favorecen la experimentación, la investigación y el descubrimiento del medio, y los materiales de desarrollo motor, para destrezas de locomoción y manipulación. 3) La Unidad didáctica, que parte de las necesidades e intereses del niño. Aqui se presentarán los "aspectos del desarrollo" perceptivo motor, socioafectivo, lenguaje, creativo e intelectual y los contenidos o áreas de formación: ciencias naturales, sociales, salud, educación física, educación estética, educación religiosa, educación sexual, lectoescritura y matemáticas. Se afirma que esta forma de trabajo facilitará la vinculación con la educación primaria. 4) Las Actividades en grupo, en donde se habla de juego-trabajo. Su diferencia con el juego libre es que los productos de estas actividades son utilizables. Se aconseja que todas las actividades ocupen el mismo tiempo que las otras.

Este currículo impactó la educación preescolar, especialmente en cuanto a las unidades didácticas. Habria que preguntarse sobre el juego, pues parecería que en su desarrollo las actividades pierden el carácter de juego libre y terminan ligadas a aprendizajes específicos. El documento reconoce que deben tenerse en cuenta las características y necesidades del niño, aunque no se desprende de una visión preparatoria para la escuela.

También en el ICBF se realizaron documentos curriculares sobre educación inicial, y aunque no se llamaron así lo son en cuanto son prescripciones para el trabajo en las aulas. En 1985, casi que paralelamente al currículo del Ministerio, el ICBF publica un documento titulado "Guía de trabajo en las unidades de protección y atención al niño" dentro del proyecto de Atención Integral al niño menor de 7 años. Aquí serán centrales las relaciones con la familia y la comunidad. Las orientaciones para el trabajo con los niños están planteadas por etapas del desarrollo y por su actividad fundamental: Primera edad (0 a 1 año): relación emocional con el adulto. Infancia temprana (1 a 3 años): relación con los objetos. Edad preescolar (3 a 7 años): el juego de roles organizado. Aquí no se hablará de "aspectos" del desarrollo, sino de "relaciones": 1) en relación con los demás; 2) en relación 
consigo mismo y conocimiento de sus posibilidades y 3) en relación con el mundo que lo rodea. El documento plantea para cada etapa unos propósitos y unas sugerencias de lo que se debe hacer.

En el año de 1990 se elabora "El Proyecto Pedagógico Educativo Comunitario", en donde, además de lo anterior, se encuentra un elemento central que es la "rutina". En ella, luego de la bienvenida están los "vamos" a: explorar, jugar, crear, comer, casa. En estos documentos que parecen tener un carácter menos primarizante, pues no aparecen las áreas de contenido, se encuentra explícito el objetivo de preparar para la primaria.

Esta situación de no coordinación entre las dos instituciones llevará a que se termine generalizando la idea de que hay un currículo para las instituciones educativas, orientadas por el Ministerio de Educación, que serán los jardines públicos y los jardines privados, y otro para las instituciones de bienestar, orientadas a los sectores pobres de la población. Lo anterior pudo llevar al "imaginario" que ya se mencionó: ¿la mirada de potenciamiento de desarrollo es para los pobres y la primarización es para las clases medias y altas?

Luego, con la Constitución de 1991 se da la obligatoriedad de un grado de preescolar (en principio el Grado 0 y luego Grado de transición). Se publicaron tres documentos en el Ministerio de Educación: la Propuesta curricular para el grado 0 , que es el documento general y continúa hablando de aspectos del desarrollo. En cuanto a las formas de trabajo estará el juego y la propuesta de trabajo por proyectos, el cual se llamó "proyecto pedagógico". Los otros dos documentos son: uno para la construcción de la lengua escrita y el otro sobre el conocimiento matemático.

En 1996 surge la Resolución 2343, atendiendo al artículo 78 de la ley 115 de 1994 que ordena que haya lineamientos generales de los procesos curriculares e indicadores de logros curriculares (León, 1997, p. 33). Sobre los lineamientos generales el artículo 3 dice que "constituyen orientaciones para que las instituciones educativas del país ejerzan la autonomía para adelantar el trabajo permanente en torno a los procesos curriculares y al mejoramiento de la calidad de la educación". Alli se formulan los Indicadores de logro para los tres grados de Preescolar. En 1997 entra en vigencia el Decreto 2247, que establece normas para prestación del servicio del nivel de preescolar. Y en 1998, se publica el Lineamiento pedagógico para la educación preescolar, donde no se hablará de currículo a pesar de ser un documento de la serie Lineamientos curriculares. En estos dos documentos se habla de Dimensiones.

\section{El auge de la educación inicial}

Ahora bien, a partir del Simposio Mundial de Educación Parvularia o Inicial, en Santiago de Chile en el año 2000, se da una mayor preocupación por la educación de los niños de la primera infancia y se generalizará el concepto de Educación Inicial, superando el de educación preescolar. Las declaraciones son explícitas en cuanto a que la educación inicial tiene identidad propia y su función no es la preparación para la escuela.

En el país, el Ministerio se concentra en la articulación transición - primaria. El ICBF ya no maneja los jardines de forma directa, sino que estos son operados por asociaciones de padres $u$ otras entidades, aunque permanece el Proyecto Pedagógico Educativo Comunitario.

Aqui es obligatorio ubicarse en Bogotá, donde el Departamento Administrativo de Seguridad Social (DABS) - hoy Secretaria Distrital de Integración Socialen los primeros años del 2000 continúa operando sus propios jardines e inicia un proceso de educación inicial "contratada" especialmente con las cajas de compensación familiar, y publica en el 2003: "Desarrollo infantil y Educación Inicial. Avances de proyecto pedagógico del DABS". Este es un documento estructurado, con una apuesta curricular, que fue la base para elaborar el Lineamiento Pedagógico Curricular para la Educación Inicial en el Distrito.

En el año 2004 la Secretaria de Educación elabora una prueba denominada: Evaluación Censal de Competencias en el Grado obligatorio de preescolar: Transición. Dicha evaluación buscaba "arrojar información sobre el estado de las competencias básicas en las áreas de lenguaje, matemáticas, ciencias naturales y valores cívicos" (p. 5). Esta evaluación se aplicó a todos los niños de 5 años a su ingreso al grado de transición en el año 2004, afortunadamente no se siguió aplicando debido a la llegada a Bogotá de 
las alcaldias de "izquierda". Esta prueba, con el argumento de la articulación entre los diferentes niveles educativos, "baja", sin más mediación que la prueba misma, las áreas obligatorias de la educación básica al grado de transición. La prueba se hace sobre las competencias para el grado primero y no tiene en cuenta lo construido para la educación inicial.

No hay que olvidar que durante este periodo, la primera década del 2000, la gran mayoría de países de América Latina elaboraron documentos curriculares para la educación inicial. En Colombia se elabora la Política Pública para la Primera Infancia, Conpes 109 del 2007, y en el 2010 se publica el documento "Desarrollo Infantil y Competencias en la Primera Infancia", que, sin quitarle su valor como documento sobre desarrollo infantil, trae el discurso de "competencias" a la primera infancia, a pesar de la connotación que tienen estas en la educación obligatoria. Paralelamente, Bogotá publica el Lineamiento Pedagógico Curricular.

\section{Algo sobre los currículos en acción de la educación inicial}

Aqui se reafirma la idea ya expuesta de que los curriculos de la educación inicial del país son fuertemente primarizantes, aunque el discurso sea de potenciamiento del desarrollo. Desde luego, esta aseveración requeriría una investigación más profunda y desafortunadamente no la hay, sin embargo, observaciones sistemáticas permiten afirmar lo anterior. Como ejemplo podemos citar los libros de texto que, como se vio, al decir de Gimeno, son currículo diseñado. Y si de algo está llena la educación inicial es de estos materiales. Los textos tienen una situación particular: se hacen para apoyar los currículos prescritos pero, a su vez, ellos crean currículo.

Tal vez la investigación que evidencia esta afirmación con mayor fuerza es la de los problemas de enseñanza de las maestras principiantes (Fandiño y Castaño, 2014) realizada entre los años 2006 y 2008. En ella se encontró que la gran mayoría de instituciones privadas donde trabajaban las egresadas era primarizante, incluso se concluyó que hay un currículo generalizado: a los tres años se aprenden las vocales, a los cuatro aparecen algunas consonantes, a los cin$c o$, por lo general, se enseña a leer y escribir. Con las matemáticas pasa algo similar: los niños de tres años deben aprender los números hasta cinco (5), los de cuatro hasta diez (10 o 20) y los de cinco hasta cien (100). Incluso si miramos las cartillas que se usan en estos grados esto es lo que se encuentra. También, se observó la prevalencia del uso de guías, de actividades de mesa y de lápiz y papel, la casi exclusividad de actividades dirigidas por la maestra donde todos los niños hacen lo mismo al mismo tiempo. Asimismo, el juego libre -donde se realiza el juego simbólico- tiene muy poco lugar en las instituciones.

Es necesario decir que en múltiples observaciones se han encontrado instituciones públicas y privadas que trabajan con diversidad de clases sociales, con miradas y prácticas centradas en el potenciamiento del desarrollo, en la escucha, en las particularidades de los niños, de sus actuaciones, y en estas se busca complejizarlas y enriquecerlas con clara intención de que los niños avancen, aunque no es menos cierto que reciben una fuerte presión para enfocarse en la primarización.

\section{La construcción de un lineamiento curricular}

En el año 2008, la Subdirección de Infancia de la Secretaría Distrital de Integración Social -SDIS (antiguo DABS) - buscó a la Universidad Pedagógica Nacional para apoyar, por medio de un convenio, la construcción de un lineamiento curricular para la ciudad de Bogotá. El convenio incluía la orientación del trabajo pedagógico de los jardines infantiles de la sDIs, por lo que la elaboración del documento estuvo alimentada por el trabajo pedagógico con los jardines. La Secretaría de Educación se sumó a la preparación del documento.

Recordemos que la sDis tenía el documento titulado: "Desarrollo Infantil y Educación Inicial", en donde se planteaban las dimensiones del desarrollo, y como estrategias de trabajo: el juego, el arte, la literatura y los escenarios de aprendizaje. Así mismo, estaba la Política Pública de Infancia y Adolescencia de Bogotá, que coincidia en plantear estas estrategias como elementos fundamentales de la educación inicial. Desde el principio se convino que era necesario agregar la "Exploración del medio". 
Entre las actividades preparatorias para la elaboración del lineamiento estuvo la observación de jornadas en los jardines y, en ese sentido, la observación de los currículos en acción. Además, se realizó la lectura de tres autores que permitirian tener como equipo una base común para abordar un lineamiento que superara la mirada primarizante y se planteara en una perspectiva, que se llamó "potenciamiento del desarrollo", entendida como dar al niño y la niña lo que necesita de acuerdo a las características de su edad para que sus capacidades se desplieguen, dándole a su vez tiempo para crecer, y que comparten los tres autores: Elkind (2004) que analiza dos conceptos: el de adelantamiento y el de educación errónea. El primero tiene la idea de esperar o exigir que los niños crecieran con rapidez para que puedan desenvolverse sin la ayuda de los adultos; el segundo, que implica darles una ventaja que los hará más brillantes y más hábiles que sus competidores. Zabalza (2008) habla de la necesidad de que en la escuela infantil exista un currículo como proyecto formativo, cuyos tres principales retos son: 1) El desarrollo de estructuras psicológicas básicas de los niños/as, referidas a: la vitalidad y a la seguridad y confianza en sí mismos. 2) El desarrollo de las capacidades genéricas: la expansión experiencial y la estimulación cognitiva y 3) La iniciación al proceso de alfabetización cultural.

Finalmente, Frabboni (1997), cuyas obras se centrarán en la identidad de la nueva escuela infantil y su currículo, propone 5 ideas fundamentales: 1) La colegialidad didáctica entre el colectivo de profesores, para construir y a la vez reflexionar sobre su trabajo pedagógico. 2) Una escuela abierta, donde el ambiente es el primer libro de lectura. Este ambiente antropológico, cultural, social y de valores es en el que se desenvuelven los niños y donde están los signos-señales, los códigos del conocimiento y la fantasía. 3) Una escuela fundada sobre la programación con intencionalidad pedagógica, que trate de reducir al máximo las desventajas culturales de los niños, en oposición a una escuela de la espontaneidad. 4) Una escuela que "teorice" sobre la posibilidad de proyectar el itinerario formativo de los niños, con hipótesis de trabajo que tengan en cuenta las necesidades reales de los niños y la comunidad. 5) Una escuela experimental que construye un currículo propio que, aunque reconoce la herencia de propuestas estructuradas, elabora estrategias didácticas flexibles y abiertas a multiplicidad de colaboraciones internas y externas.

El lineamiento se discutió con las universidades y con grupos de maestras, y se profundizó el papel de los pilares de la educación inicial: juego, arte, literatura y exploración del medio, como las actividades principales de la relación adulto-niño, que ya venían siendo planteadas. Se insistió en que ellas son actividades que, por sí mismas, potencian el desarrollo de todas las dimensiones, en la medida en que se programan y se diseñan ambientes que las posibilitan y les dan todo su valor.

Luego, con la estrategia De Cero a Siempre el Gobierno Nacional, a través del MEN, publica los Referentes Técnicos de la Educación Inicial (2012), en los que se desarrollan con mayor profundidad los cuatro pilares y serán nombrados como actividades rectoras. En ellos se sustenta el hecho de que un niño que juega libremente, además de elaborar comprensiones sobre el mundo adulto, desarrolla su pensamiento simbólico, su lenguaje, y se enfrenta con situaciones que le permiten aprender a escuchar al otro, a respetarlo. Que el arte le permite la expansión de sus capacidades expresivas y creativas donde todo su cuerpo está presente. Que la literatura le permite elaborar sus emociones, además de acercarlo a la lengua oral y escrita, enriquecer su imaginación y su pensamiento. Que la exploración del medio o del ambiente físico, social y cultural, lo inicia en la comprensión y estructuración del mismo. En este último, se presentan los proyectos de aula, que parten de preguntas de los niños, en particular de los de 4 y 5 años, para iniciarse en la alfabetización cultural. En este sentido, si se diseñan ambientes y se potencian las interacciones con los otros y con el mundo, un niño está listo para ingresar a la educación primaria con ganas de seguir aprendiendo, que es lo más importante.

Pero para que esto se realice de modo satisfactorio es indispensable la colegialidad, el trabajo conjunto de maestros. Pocos conocen y saben más de las necesidades de los niños que los maestros. Dejemos entonces que sus saberes se visibilicen: reflexionemos sobre ellos, enriquezcámoslos. Son los grupos de 
maestras, con condiciones adecuadas, con acompañamientos dialógicos, quienes pueden construir los mejores currículos para la educación de los niños de la primera infancia, incluso, para que este enfoque centrado en el niño se amplíe al primer ciclo de la básica, es decir, a los niños hasta los 8 años. Posteriormente, se han elaborado otros documentos que buscan precisar más el currículo prescrito de la Educación Inicial y el debate continúa.

\section{Referencias}

Aristizábal, M. (2012). La irrupción de la teoría curricular a partir de 1960 y su influencia en las reformas educativas en Colombia. Acción pedagógica, 21 (1), 28-37.

Colmenar, C. (1995). Génesis de la educación infantil en la Sociedad Occidental. Revista complutense de educación, $6(1), 15-29$.

Departamento Administrativo de Bienestar Social (DABS). (2003). Desarrollo Infantil y Educación Inicial. Autor.

Díaz Barriga, Á. (2003). Currículum. Tensiones conceptuales y prácticas. Revista Electrónica de Investigación Educativa, 5 (2). http://redie.uabc.mx/vol5no2/contenido-diazbarriga.html

Elkind, D. (2004). La educación errónea. Fondo de Cultura Económica.

Fandiño, C. y Castaño I. (2014). Haciéndose maestras. UPN, CIUP, Magisterio.

Frabboni, F. (1997). La educación del niño de 0 a 6 años. Cincel.

Gimeno, J. (1998). El curriculum: una reflexión sobre la práctica. Morata.

ICBF. (1985). Guía de trabajo en las unidades de atención y protección al niño. Autor.

ICBF. (1990). Proyecto Pedagógico Educativo Comunitario. Autor.

León, T. (1997). Indicadores. Un mirador para la educación. Norma.

Luzuriaga, L. (1943). La Educación Nueva. Universidad Nacional de Tucumán.

Michelet, A. (1988). Los útiles de la infancia. Herde.

Ministerio de Educación Nacional, MEN. (1984). Currículo de Preescolar: niños de 4 a 6 años. Autor.

Ministerio de Educación Nacional, MEN. (1996). Hacia la comprensión del nivel de Educación preescolar. Autor.

Ministerio de Educación Nacional, MEN. (1998). Lineamiento pedagógico para la educación preescolar. Autor.

Ministerio de Educación Nacional, MEN. (2010). Desarrollo Infantil y Competencias en la Primera Infancia. Autor.
Ministerio de Educación Nacional, MEN. (2012). De cero a siempre. El arte en la educación inicial. Documento No. 21. Autor.

Ministerio de Educación Nacional, MEN. (2012). El juego en la educación inicial. Documento No. 22. Autor.

Ministerio de Educación Nacional, MEN. (2012). La literatura en la educación inicial. Documento No. 23. Bogotá, Colombia. Autor.

Ministerio de Educación Nacional, MEN. (2012). La exploración del medio en la educación inicial. Documento No. 24. Autor.

Sanchidrian, C. y Ruiz, J. (2010). Historia y perspectiva actual de la educación infantil. Graó.

Sanchidrian, C. (2010). La escuela maternal francesa. La construcción de un modelo propio. En C. Sanchidrian y J. Ruiz (Coord.), Historia y perspectiva actual de la educación infantil (pp. 69-90). Graó.

Vasco, C. (2011). La presencia de Piaget en la educación coIombiana 1960-2010. Revista Colombiana de Educación, 60, 15-40. https://doi.org/10.17227/01203916.836

Zabalza, M. (2003). El currículo de la Educación Infantil. http://www.infantiae.org/zabacurrilTA1807.asp 


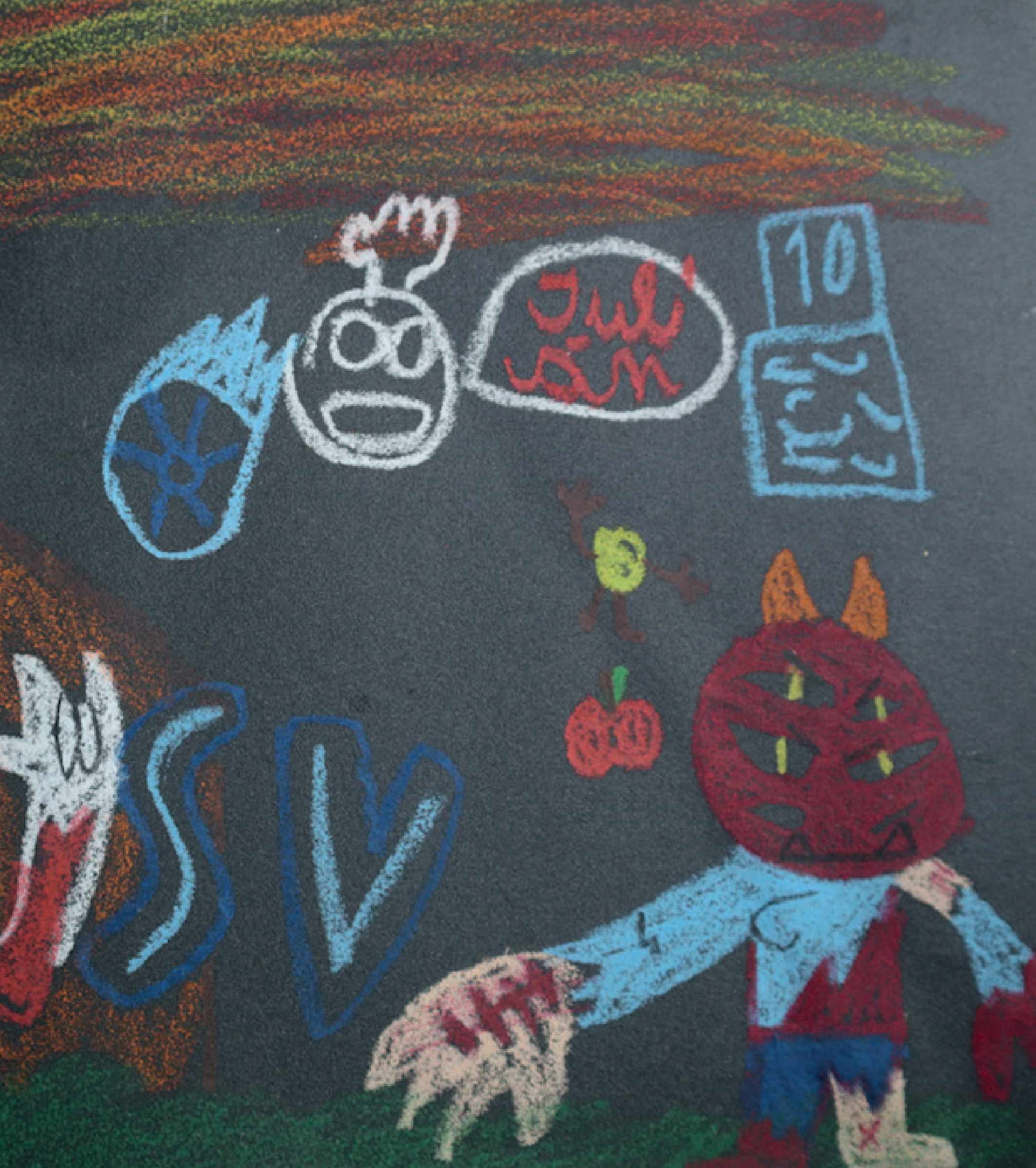

